\title{
Optimal sap flux sensor allocation for stand transpiration estimates: a non-dimensional analysis
}

\author{
Hikaru Komatsu $^{1,2} \cdot$ Tomonori Kume ${ }^{3}$ Yoshinori Shinohara ${ }^{4,5}$
}

Received: 12 December 2016/Accepted: 11 April 2017 / Published online: 26 April 2017

(C) INRA and Springer-Verlag France 2017

\begin{abstract}
- Key message Measuring between-tree variations in sap flux density rather than azimuthal variations should be prioritized for reliable stand transpiration estimates based on sap flux methods.

- Context Stand transpiration (E) estimated using sap flux methods includes uncertainty induced by azimuthal variations and between-tree variations in sap flux density $(F)$.

- Aims This study examines whether or not measuring $F$ for two or more azimuthal directions to cover azimuthal variations in $F$ leads to more reliable $E$ estimates. This examination was done under the assumption that azimuthal and between-
\end{abstract}

Handling Editor: Erwin Dreyer

Contribution of the co-authors Kume: theorization and editing the paper.

Shinohara: raw data preparation and editing the paper.

Hikaru Komatsu

kmthkr@gmail.com

Tomonori Kume

kumett@ntu.edu.tw

Yoshinori Shinohara

shinohara@cc.miyazaki-u.ac.jp

1 The Hakubi Center for Advanced Research, Kyoto University, Kyoto 606-8302, Japan

2 Graduate School of Agriculture, Kyoto University, Kyoto 606-8502, Japan

3 School of Forestry and Resource Conservation, National Taiwan University, Taipei 106, Taiwan

4 Faculty of Agriculture, Kyushu University, Fukuoka 812-8581, Japan

5 Faculty of Agriculture, University of Miyazaki, Miyazaki 889-2192, Japan tree variations in $F$ are not systematic and when a limited number of sensors are available.

- Methods We first non-dimensionalized the theoretical framework established by a previous study and developed a general hypothesis. We then validated the hypothesis quantitatively by numerical experiments.

- Results The non-dimensionalized theory allowed us to hypothesize that measuring $F$ for one azimuthal direction would reduce uncertainty in $E$ estimates more effectively than measuring $F$ for two or more azimuthal directions. Results of the numerical experiments were found to support this hypothesis. - Conclusion When the aforementioned assumptions are satisfied, allocating sensors to measure $F$ for one azimuthal direction to cover between-tree variations in $F$ always leads to more reliable $E$ estimates.

Keywords Azimuthal variations · Between-tree variations . Observation design $\cdot$ Sap flux methods $\cdot$ Scaling up · Uncertainty

\section{Introduction}

Estimating forest transpiration $(E)$ is an important topic in forestry, ecology, and hydrology (Ewers et al. 2011; Kume et al. 2011). $E$ affects the amounts of other components of the forest water cycle, such as soil water content, deep percolation, and catchment runoff (Vertessy et al. 2001; Shinohara and Otsuki 2016). $E$ also relates with forest carbon absorption through stomatal gas exchanges and then relates with other components of the forest carbon cycle (Running and Coughlan 1988; Landsberg and Waring 1997).

Sap flux methods (Granier 1987; Swanson 1994) are promising methods of estimating $E$ based on field measurements. Sap flux methods, unlike micrometeorological methods, are 
applicable to forests located on complex terrain (Kumagai et al. 2007; Kume et al. 2016). E estimated using sap flux methods is less seriously subject to errors associated with the scaling-up process than that estimated using porometry (Leverenz et al. 1982; Ewers and Oren 2000). Because of these advantages, sap flux methods are widely applied to estimate $E$. This is particularly the case for even-aged monospecific stands because of the simplicity of scaling-up from sensors to a tree and then from trees to a stand for such stands (Delzon and Loustau 2005; Komatsu et al. 2014).

Even when the method is applied to an even-aged monospecific stand, there is uncertainty in $E$ estimates made using sap flux methods. This uncertainty is partly due to variations in sap flux density $(F)$ within a tree (i.e., radial and azimuthal variations) and those between trees (Köstner et al. 1996; Shinohara et al. 2013; Moon et al. 2015). This uncertainty is expected to decrease with an increase in the number of sensors installed in the stand. However, the number of sensors available for measurements of $F$ is usually limited. This is particularly the case when conducting sap flux measurements of $E$ simultaneously at several forest sites with different stand properties (e.g., species, leaf area, stem density, and canopy height) and/or with different treatments (e.g., irrigation, fertilization, and $\mathrm{CO}_{2}$ enrichment), as done by many previous studies (Alsheimer et al. 1998; Ewers et al. 2005). It is therefore important to allocate sensors effectively to reduce uncertainty in $E$ estimates.

To reduce uncertainty in $E$ estimated using a limited number of sensors, it is effective to model systematic variations in $E$ within and between trees and use the model in estimating $E$. This strategy is commonly used to reduce the number of sensors allocated radially to consider radial variations in $F$, because radial variations in $F$ are usually systematic (Ford et al. 2007; Poyatos et al. 2007; Bosch et al. 2015). For example, Ford et al. (2007) determined the typical profile of radial variations in $F$ based on preliminary measurements of $F$ for several trees in a Pinus strobus L. stand. Applying the profile to the other trees in the stand and measuring $F$ for the outmost xylem band of the trees in the stand, Ford et al. (2007) estimated transpiration for individual trees and then $E$.

In contrast with radial variations in $F$, azimuthal variations in $F$ are not particularly systematic. Many studies measuring azimuthal variations in $F$ neither observed higher/lower $F$ for a specific azimuthal direction ( $\mathrm{Lu}$ et al. 2000; Cohen et al. 2008; Tateishi et al. 2008; Sato et al. 2012) nor identified factors explaining the variations well (Loustau et al. 1998; Sato et al. 2012). Although several studies (Tsuruta et al. 2010; Shionohara et al. 2013; Moon et al. 2015) reported higher/lower $F$ for a specific azimuthal direction, the difference in $F$ according to azimuthal direction was less systematic than that according to depth (i.e., radial variations). Only a few studies (Oren et al. 1999) reported more pronounced differences in $F$ according to the azimuthal direction than those according to depth.
Between-tree variations in $F$ are not particularly systematic for many cases, although they are systematic for some cases. Previous studies examined relationships of $F$ representative of a tree with structural parameters (e.g., stem diameter and tree height). Many of the studies (Köstner et al. 1996; Oren et al. 1998; Pataki et al. 2000; Kume et al. 2010a; Shinohara et al., 2013) reported poor correlation between $F$ and structural parameters. However, several studies have reported strong correlation between $F$ and a structural parameter (Granier et al. 2000; Kume et al. 2012). In such cases, it might be more efficient to classify trees according to the value of the parameter and develop a model for the relationship between the structural parameter and $F$ (see discussion in Komatsu et al. 2016).

When azimuthal and between-tree variations in $F$ are not systematic and when a limited number of sensors are available, what is the optimum sensor allocation? Which leads to more reliable $E$ estimates, measuring $F$ for two or more azimuthal directions to cover azimuthal variations in $F$ or measuring $F$ for only one azimuthal direction to cover betweentree variations in $F$ more broadly? Although there would have been researchers who faced this question when they started measurements of $F$ for $E$ estimates using sap flux methods, very few have examined this question explicitly. Our previous study (Komatsu et al. 2016) is possibly the only exception. Komatsu et al. (2016), inspired by several previous studies (Kume et al. 2012; Shinohara et al. 2013), theorized the question statistically and identified the conditions under which measuring $F$ for two or more azimuthal directions reduces uncertainty in $E$ estimates more effectively than measuring $F$ for one azimuthal direction when the number of sensors is limited. Komatsu et al. (2016) recommended researchers to make preliminary measurements of $F$ for the target site to examine whether the conditions are satisfied and to determine the optimal sensor allocation before initiating full measurements.

This study, however, demonstrates that the conditions are never satisfied, i.e., measuring $F$ for one azimuthal direction is always found to allow more reliable $E$ estimates within the framework of the theory. It is thus unnecessary to examine whether the conditions are satisfied based on preliminary measurements of $F$ for the target site. The approach taken in the present paper comprises three steps. First, we transform the theory developed in our previous study to a non-dimensional form, which allows us to examine the aforementioned question in a comprehensive way. We then make a qualitative analysis using the transformed theory to develop a hypothesis that measuring $F$ for two or more directions to cover azimuthal variations in $F$ would never lead to more reliable $E$ estimates when azimuthal and between-tree variations in $F$ are not systematic and when a limited number of sensors are available. We finally conduct numerical experiments based on the theory to confirm the validity of our hypothesis. 


\section{Transformation of theory}

This section first describes the original theory developed by Komatsu et al. (2016) and then transforms it to a nondimensional form. This section finally clarifies the conditions under which measuring $F$ for two or more azimuthal directions leads to more reliable $E$ estimates.

\subsection{Original theory}

Suppose that there are $N$ trees in a stand, that $n$ sensors are available, and that the sensors are allocated to measure $F$ in $m$ azimuthal directions of each tree. We would be able to measure $F$ for $n / m$ trees, when we assume that one sensor is required to obtain $F$ representative of an azimuthal direction. Note that when $n / m$ is not an integer, the number of trees on which we would be able to measure $F$ is the maximum integer satisfying $<n / m$. The assumption that one sensor is required to obtain $F$ representative of an azimuthal direction is valid when one sensor covers the sapwood depth (Kume et al. 2010b; Komatsu et al. 2010, 2012) or when $F$ for inner xylem bands is estimated from $F$ recorded at the outmost xylem band using the sensor (Ford et al. 2007; Poyatos et al. 2007; Bosch et al. 2015). In the theoretical framework, we assume that azimuthal and between-tree variations in $F$ are not systematic. The framework is actually applicable to the case with azimuthal variations in $F$ being systematic, if we assume that the azimuthal directions for $F$ measurements are randomly selected.

$E$ on a ground-area basis is equal to the sum of transpiration for the $N$ trees in the stand divided by the ground area of the stand $(S)$ :

$E=\frac{\sum_{i=1}^{N} Q_{i}}{S}$,

where $Q_{i}$ is transpiration for the $i$-th tree calculated as the product of the sap flux per unit sapwood area representative of the tree calculated from $F$ in $m$ azimuthal directions $\left(<F>_{i, m}\right)$ and the sapwood area of the tree $\left(a_{i}\right)$ :

$E=\frac{\sum_{i=1}^{N}<F>_{i, m} \cdot a_{i}}{S}$

Note that the brackets of $\langle F\rangle_{i, m}$ are added to denote that the sap flux density represents that at a tree scale, not at a sensor scale (Fig. 1). $E$ is then rewritten as

$E=\frac{1}{N} \cdot \sum_{i=1}^{N}\left(\frac{N}{S} \cdot\left\langle F>_{i, m} \cdot a_{i}\right)\right.$,

where $(N / S) \cdot\langle F\rangle_{i, m} \cdot a_{i}$ denotes the stand transpiration calculated assuming that the transpiration for each of $N$ trees is equal to that for the $i$-th tree. Equation (3) indicates that $E$ is the arithmetic mean of $(N / S) \cdot\langle F\rangle_{i, m} \cdot a_{i}$ for the $N$ trees. $\langle F\rangle_{i, m}$ is not available for all $N$ trees, but is available for $n /$ $m$ trees. We therefore approximate Eq. (3) by

$E=\frac{1}{n / m} \cdot \sum_{i=1}^{n / m}\left(\frac{N}{S} \cdot\left\langle F>_{i, m} \cdot a_{i}\right)\right.$

where we assume that the arithmetic mean of $(N / S) \cdot\langle F\rangle_{i, m} \cdot a_{i}$ for the $N$ trees is equal to that for $n / m$ trees whose $\langle F\rangle_{i, m}$ is available. The inaccuracy introduced by this approximation reduces with increasing $n / m$. This is similar to the case when using an equation expressing $E$ as a product of the mean sap flux and the sapwood area on a plot scale (Granier et al. 1996; Ewers and Oren 2000).

$\langle F\rangle_{i, m}$ is then approximated by the arithmetic mean of $F$ for $m$ azimuthal directions

$<F>_{i, m}=\frac{1}{m} \cdot \sum_{j=1}^{m} F_{i, j}$,

where $F_{i, j}$ is $F$ for the $j$-th azimuthal direction of the $i$-th tree. Equations (4) and (5) are the basic equations for $E$ estimates. Equations (4) and (5) correspond to scaling up from trees to the stand and from sensors to a tree, respectively. We here find a trade-off that a greater $m$ value leads to less accurate approximation of Eq. (4) (i.e., treeto-stand scaling up) but more accurate approximation of Eq. (5) (i.e., sensor-to-tree scaling up). For example, if 100 sensors are available and all sensors are installed on one tree in the stand, the approximation of Eq. (4) would be quite inaccurate while that of Eq. (5) would be very accurate. This trade-off relation determines whether or not measuring $F$ for two or more azimuthal directions leads to more reliable $E$ estimates.

Note that $a_{i}$ is assumed to be known for all trees in this theory. This assumption would be reasonable for two reasons. First, measurements of sapwood areas do not usually require as many resources as those of sap flux (Kume et al. 2010a; Macfarlane et al. 2010). Second, even when sapwood areas for merely a limited number of trees are available, sapwood areas for the other trees can be estimated relatively easily and accurately using allometric equations (Bond-Lamberty et al. 2002; Kumagai et al. 2005).

\subsection{Transformation of the theory}

We here transform the basic equations for $E$ estimates (i.e., Eqs. (4) and (5)) into non-dimensional equations, which are simpler than the original ones but mathematically equivalent to them. For non-dimensionalization of Eq. (4), we first introduce two variables defined by

$\overline{<F}_{i, m=\infty}=\frac{1}{N} \cdot \sum_{i=1}^{N}\left\langle F>_{i, m=\infty}\right.$, 
Fig. 1 Schematic drawing of how the distribution of nondimensionalized stand transpiration $\left(E^{*}\right)$ estimates is produced from sensor-scale sap flux density $\left(F^{*}{ }_{i, j}\right)$
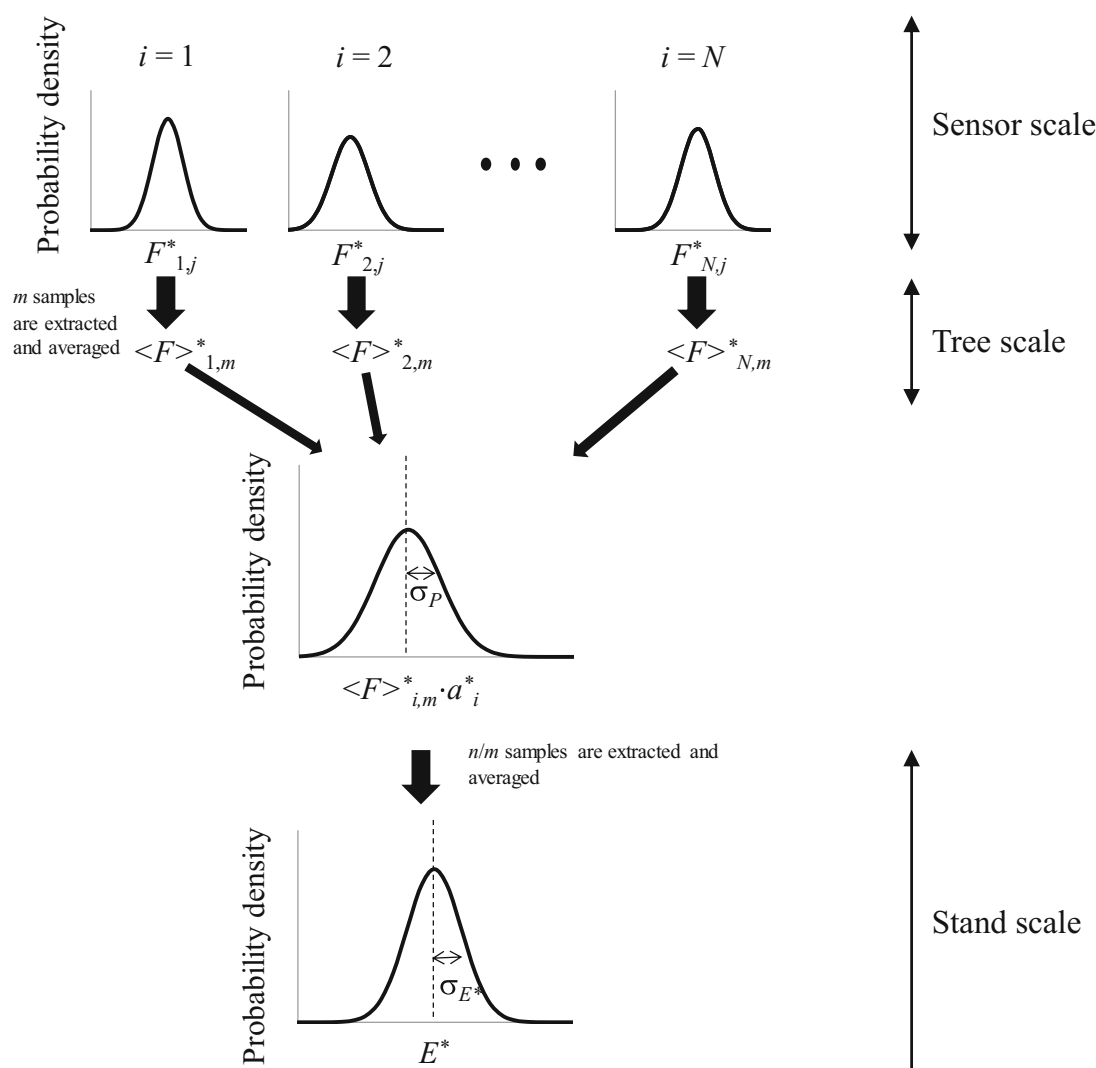

$\bar{a}=\frac{1}{N} \cdot \sum_{i=1}^{N} a_{i}$

$<F>^{*}{ }_{i, m}=\frac{\left\langle F>_{i, m}\right.}{\left\langle F>_{i, m=\infty}\right.}$,

where $\langle F\rangle_{i, m=\infty}$ is the ideal limit of $\langle F\rangle_{i, m}$ when $m$ approaches infinity. Note that $\langle F\rangle_{i, m}=\infty$ is a term introduced merely for non-dimensionalization. $m$ cannot exceed $n$ in an actual experimental setting, which does not affect our conclusions. $\langle F\rangle_{i, m=\infty}$ is thus equal to the mean value of the distribution for $F_{i, j}$. If we divide Eq. (4) by $(N / S) \cdot \overline{\langle F\rangle}_{i, m=\infty} \cdot \bar{a}$, we obtain

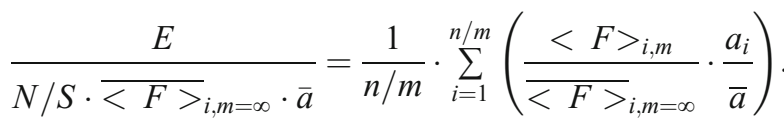

This equation can be rewritten as

$E^{*}=\frac{1}{n / m} \cdot \sum_{i=1}^{n / m}\left(\langle F\rangle^{*}{ }_{i, m} \cdot a^{*}{ }_{i}\right)$

This equation is the non-dimensional form of Eq. (4). Here, $E^{*},<F>^{*}{ }_{i, m}$, and $a^{*}{ }_{i}$ are given by

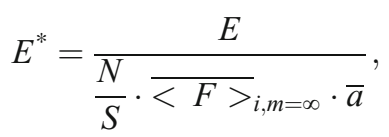

$a_{i}^{*}=\frac{a_{i}}{\bar{a}}$.

For the non-dimensionalization of Eq. (5), we divide Eq. (5) by $\overline{\langle F}_{i, m=\infty}$ and obtain

$\frac{\left\langle F>_{i, m}\right.}{\overline{\left\langle F>_{i, m=\infty}\right.}}=\frac{1}{m} \cdot \sum_{j=1}^{m} \frac{F_{i, j}}{\overline{\left\langle F>_{i, m=\infty}\right.}}$

This equation can be rewritten as

$<F>^{*}{ }_{i, m}=\frac{1}{m} \cdot \sum_{j=1}^{m} F^{*}{ }_{i, j}$,

where $F^{*}{ }_{i, j}$ is

$F_{i, j}^{*}=\frac{F_{i, j}}{\overline{\left\langle F>_{i, m=\infty}\right.}}$.

This equation is the non-dimensional form of Eq. (5).

Equations (9) and (14) allow us to conduct numerical experiments that are simpler and more comprehensive than those conducted using Eqs. (4) and (5). Equations (9) and (14) are mathematically equivalent to Eqs. (4) and (5), but include 
fewer parameters to be determined. For example, numerical experiments using Eqs. (9) and (14) do not require determination of $N$ and $S$, while those using Eqs. (4) and (5) do require such determination.

\subsection{Conditions justifying measurements of $F$ for two or more directions}

Based on the non-dimensional equations, we here explain the conditions under which measuring $F$ for two or more azimuthal directions leads to more reliable $E^{*}$ estimates. To explain the conditions, we first define uncertainty in $E^{*}$ estimates. $E^{*}$ is estimated by scaling up from sensors to a tree (Eq. (14)) and then from trees to the stand (Eq. (9)). In the former scaling-up process, $m$ samples are randomly extracted from the distribution of $F_{i, j}^{*}$ (distributions at the top of Fig. 1) to determine $\left\langle F{ }^{*}{ }_{i, m}\right.$ using Eq. (14). In the second scaling-up process, values of $\langle F\rangle_{i, m}^{*}$ for all $N$ trees are collated to obtain the distribution for $\left\langle F>_{i, m}^{*} \cdot a_{i}{ }_{i}\right.$ (the distribution in the middle of Fig. 1). $n / m$ samples are then extracted from this distribution to obtain $E^{*}$ using Eq. (9). When these processes are repeated many times, a probability distribution of $E^{*}$ (the distribution at the bottom of Fig. 1) is obtained. We define the variation in this probability distribution as the uncertainty in $E^{*}$ estimates.

According to basic statistics, the standard deviation for the mean of randomly selected samples is equal to the standard deviation of the population divided by the square root of the sample size (Fujii 2005; Peck and Devore 2011). The standard deviation for the probability distribution of $E^{*}\left(\sigma_{E^{*}}\right)$ is thus written as

$\sigma_{E^{*}}=\frac{\sigma_{P}}{\sqrt{n / m}}$,

where $\sigma_{P}$ is the standard deviation for $\left\langle F>^{*}{ }_{i, m} \cdot a_{i}{ }_{i}\right.$ of all $N$ trees in the stand. Suppose that we are comparing $\sigma_{E^{*}}$ values for $m$ being 1 and $k(\geq 2)$. According to Eq. (16), $\sigma_{E^{*}}$ for $m$ being 1 and $k\left(\sigma_{E^{*}(m=1)}\right.$ and $\sigma_{E^{*}(m=k)}$, respectively) is written as

$$
\begin{gathered}
\sigma_{E^{*}(m=1)}=\frac{\sigma_{P(m=1)}}{\sqrt{n}}, \\
\sigma_{E^{*}(m=k)}=\frac{\sigma_{P(m=k)}}{\sqrt{n / k}},
\end{gathered}
$$

where $\sigma_{P(m=1)}$ and $\sigma_{P(m=k)}$ are $\sigma_{P}$ for the cases of $m$ being 1 and $k$, respectively. From these equations, we obtain conditions for $\sigma_{E^{*}}$ with $m=k$ less than that with $m=1$ :

$\frac{\sigma_{P(m=k)}}{\sigma_{P(m=1)}}<\frac{1}{\sqrt{ } k}$.
Under these conditions, measuring $F$ for $k$ azimuthal directions on each of the $n / k$ trees instead of measuring $F$ for one azimuthal direction on each of the $n$ trees is justified.

\section{Hypothesis}

We hypothesize that Eq. (19) can never be satisfied, because we infer that even when $\sigma_{P(m=k)} / \sigma_{P(m=1)}$ is minimized, $\sigma_{P}$ $(m=k) / \sigma_{P(m=1)}$ is equal to $1 / \sqrt{ } k \cdot \sigma_{P(m=k)} / \sigma_{P(m=1)}$ is expected to be minimized when the variation in $\left\langle F{ }^{*}{ }_{i, m} \cdot a_{i}{ }_{i}\right.$ among trees (i.e., the variation of the distribution in the middle of Fig. 1) reduces most efficiently with increasing $m$, i.e., when there are variations in neither the distribution of $F_{i, m}^{*}$ nor the value of $a_{i}{ }_{i}$ among trees in the stand (Fig. 2a, b). The variation in $\langle F\rangle^{*}{ }_{i, m} \cdot a^{*}{ }_{i}$ approaches zero when $m$ approaches infinity under these conditions (Fig. 2c). In contrast with this case, the variation in $\langle F\rangle_{i, m}^{*} \cdot a^{*}{ }_{i}$ does not approach zero in a realistic case when there are variations in the distribution of $F_{i, m}^{*}$ and the value of $a_{i}{ }_{i}$ among trees (Fig. $2 \mathrm{~d}-\mathrm{f}$ ).
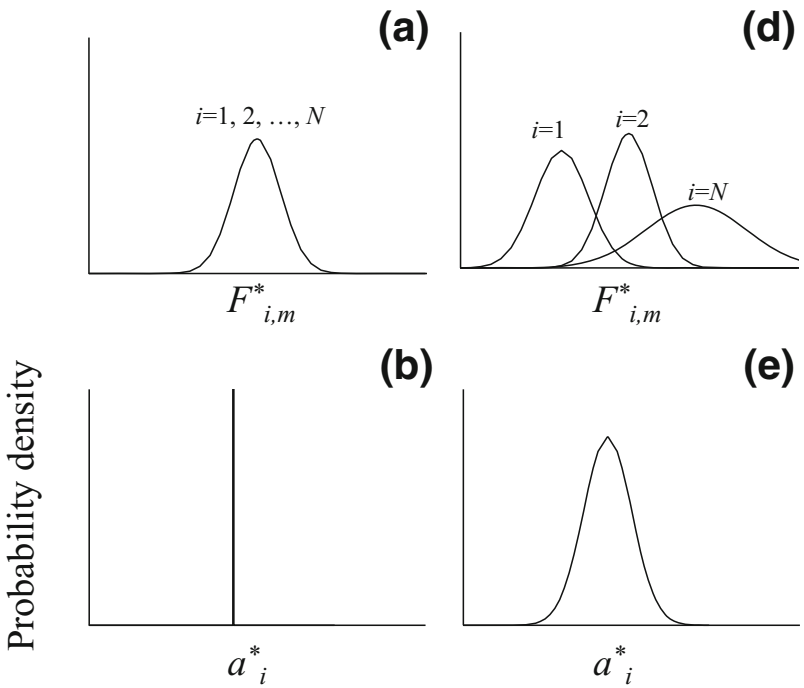

(e)
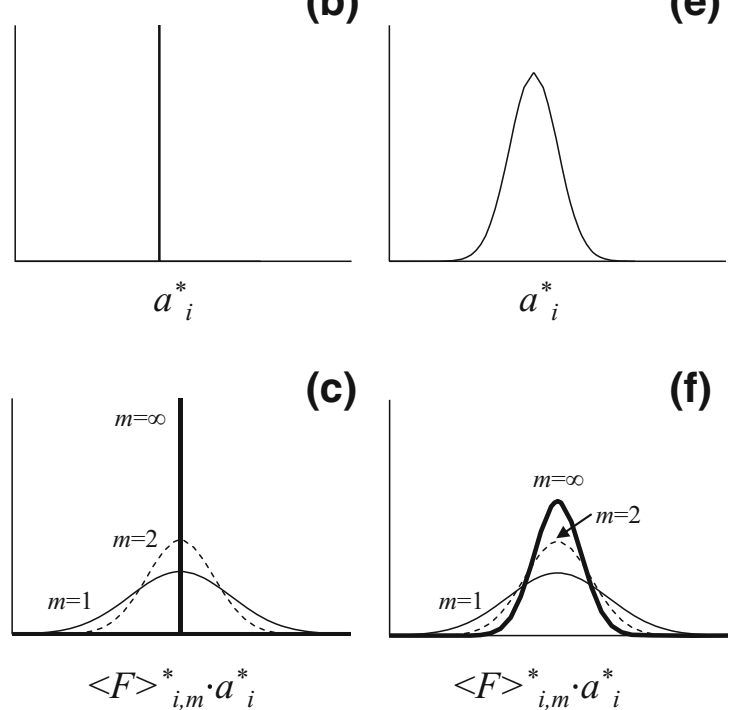

Fig. 2 a Distributions of non-dimensionalized sap flux density $\left(F^{*}{ }_{i, m}\right)$ for the $N$ trees. $\mathbf{b}$ The value of non-dimensionalized sapwood area $\left(a_{i}^{*}\right)$ among the $N$ trees. c Changes in the variation in $\langle F\rangle_{i, m}^{*} \cdot a_{i}^{*}$ with changing $m$ when there are variations in neither the distribution of $F_{i, m}^{*}$ nor the value of $a_{i}{ }_{i}$ among the trees. This is exactly the case when $\sigma_{P}$ ${ }_{(m=k)} / \sigma_{P(m=1)}$ is minimized. d-f The same as Fig. 2a-c, but for a realistic case when there are variations in the distribution of $F_{i, m}^{*}$ and in the value of $a^{*}{ }_{i}$ 
When there are variations in neither the distribution of $F_{i, m}^{*}$ nor the value of $a_{i}{ }_{i}$ among trees (Fig. 2a, b), $\sigma_{P(m=k)} / \sigma_{P(m=1)}$ is expected to be equal to $1 / \sqrt{ } k$. Under these conditions (Fig. 2a, b), extracting $m$ samples from each of the $F_{i, j}^{*}$ distributions for $n / m$ trees is completely equivalent to extracting one sample from each of the $F_{i, j}^{*}$ distributions for $n$ trees. The variation in $\langle F\rangle^{*}{ }_{i, m} \cdot a^{*}{ }_{i}$ for the former case is thus completely the same as that for the latter case. $\sigma_{P(m=k)} / \sigma_{P}$ $(m=1)$ is consequently expected to be equal to the threshold value, i.e., $1 / \sqrt{ } k$ (see Eq. (19)). In reality, the distribution of $F^{*}{ }_{i, j}$ and the value of $a_{i}{ }_{i}$ differ among trees (Fig. 2d, e), which suggests $\sigma_{P(m=k)} / \sigma_{P(m=1)}$ is always greater than $1 / \sqrt{ } k$.

\section{Methods of numerical experiments}

To examine the hypothesis described above, we calculated $\sigma_{P}$ $(m=k) / \sigma_{P(m=1)}$ based on numerically generated distributions of $\left\langle F{ }^{*}{ }_{i, m} \cdot a^{*}{ }_{i}\right.$ for $m=1,2,3$, and 4 under various conditions of $F^{*}{ }_{i, j}$ and $a_{i}{ }_{i}$. To generate distributions of $\left\langle F>^{*}{ }_{i, m} \cdot a^{*}{ }_{i}\right.$, we first produced distributions of $F^{*}{ }_{i, j}$ and $a^{*}{ }_{i}$ for many trees. Here, we set the number of trees to be 5000 , because we found no dependency of calculation results on the number when it exceeded 5000. We then extracted $m$ samples with replacement from the distribution of $F_{i, j}^{*}$ for each tree to generate the distributions of $\left\langle F>{ }_{i, m}^{*} \cdot a^{*}{ }_{i}\right.$ and to determine $\sigma_{P}$ for $m=1,2$, 3 , and 4 . Note that the number of trees assumed here (i.e., 5000 trees) is generally greater than the number of trees included in an experimental plot. However, the assumption is reasonable because researchers install an experimental plot for sap flux measurements to obtain $E$ representative not for the plot but for the stand including the plot.

The distribution of $F_{i, j}^{*}$ for each tree was assumed to follow a normal distribution (Fig. 3a). The mean value of the distribution is by definition equal to $\langle F\rangle^{*}{ }_{i, m=\infty}$. Here, $\langle F\rangle^{*}{ }_{i, m=\infty}$ is the ideal limit of the mean of $m$ samples extracted from the distribution when $m$ approaches infinity. The coefficient of variation $(\mathrm{CV})$ of the distribution was assumed to be $\mathrm{CV}_{F}$, a constant to be determined. The distribution of $\langle F\rangle^{*}{ }_{i, m=\infty}$ for the 5000 trees was further assumed to follow a normal distribution (Fig. 3b). The mean value of the distribution is, by definition, equal to 1.0 (see Eq. (11)). The CV of the distribution was assumed to be $\mathrm{CV}_{<F>}$, a constant to be determined. The distribution of $a_{i}{ }_{i}$ for the 5000 trees was assumed to follow a normal distribution (Fig. 3c). The mean value of the distribution is, by definition, 1.0 (see Eq. (12)). The CV value was assumed as $\mathrm{CV}_{a}$, a constant to be determined.

The numerical experiments examined the relationships of $\sigma_{P(m=k)} / \sigma_{P(m=1)}$ with $\mathrm{CV}_{<F>}$ and $\mathrm{CV}_{a}$. We first assumed $\mathrm{CV}_{a}$ to be zero (i.e., there was no variation in $a^{*}{ }_{i}$ among trees, as in Fig. 2b) and examined the change in $\sigma_{P(m=k)} / \sigma_{P(m=1)}$ with $\mathrm{CV}_{<F>}$ increasing from zero. According to our hypothesis, $\sigma_{P(m=k)} / \sigma_{P(m=1)}$ was expected to equal $1 / \sqrt{ } k$ only when
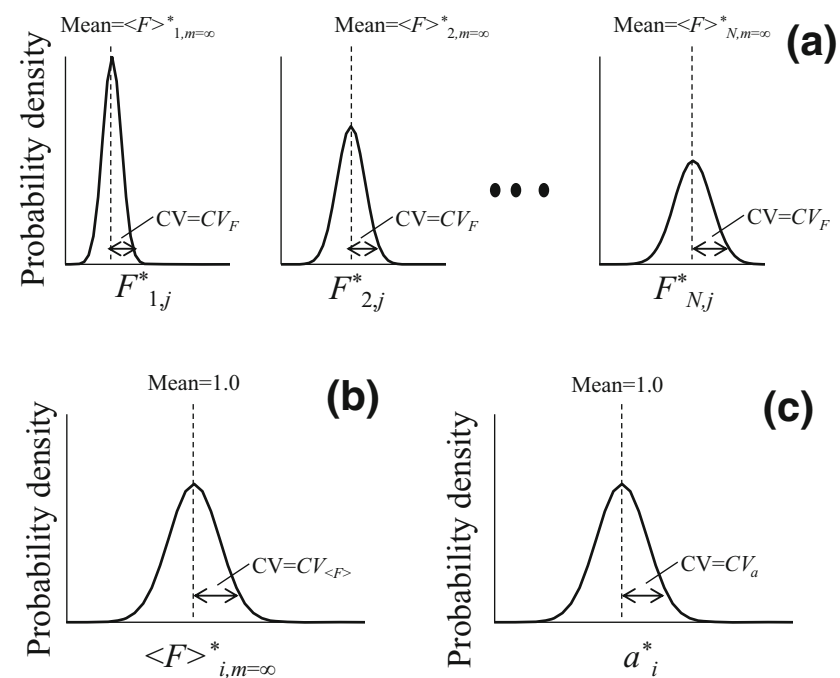

(b)

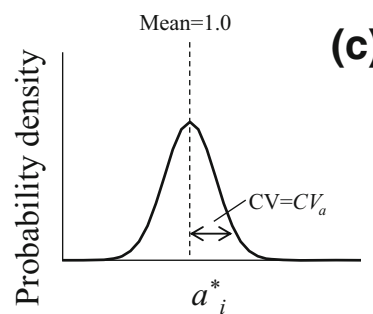

Fig. 3 Definition of the parameters used in the numerical experiments: parameters concerning a within-tree variations in non-dimensionalized sap flux density $\left(F_{i, j}^{*}\right)$, b between-tree variations in nondimensionalized sap flux density when $m$ approaches infinity $\left(<F>^{*}{ }_{i, m}=\infty\right)$, and $\mathbf{c}$ between-tree variations in non-dimensionalized sapwood area $\left(a_{i}^{*}\right)$

$\mathrm{CV}_{<F>}$ was zero, i.e., when there was no variation in the distributions of $F_{i, j}^{*}$ among trees (Fig. 2a). $\sigma_{P(m=k)} / \sigma_{P(m=1)}$ was expected to be greater than $1 / \sqrt{ } k$ otherwise. We then assumed a realistic value of $\mathrm{CV}_{a}(>0)$, i.e., $a^{*}{ }_{i}$ varied among trees (Fig. 2e). We again examined the change in $\sigma_{P}(m=k) / \sigma_{P}$ $(m=1)$ with $\mathrm{CV}_{<F>}$ increasing from zero. In this case, $\sigma_{P}$ ${ }_{(m=k)} / \sigma_{P(m=1)}$ was expected to be always greater than $1 / \sqrt{ } k$.

The parameters were determined from observation data recorded for a Cryptomeria japonica stand (Shinohara et al. 2013; Komatsu et al. 2016) in a manner detailed in the Appendix. $\mathrm{CV}_{F}$ was determined as 0.29 . The upper boundary of the change in $\mathrm{CV}_{<F>}$ was assumed as 0.80 , more than three times the value determined using the observation data $(0.24)$, to fully cover a realistic range of $\mathrm{CV}_{<F>}$. $\mathrm{CV}_{a}$ for the realistic case (i.e., when $a_{i}^{*}$ varied among trees) was determined as 0.42 .

\section{Results}

In the case of $\mathrm{CV}_{a}$ being zero in the numerical experiments, $\sigma_{P}$ $(m=k) / \sigma_{P(m=1)}$ equaled $1 / \sqrt{ } k$ only when $\mathrm{CV}_{<F>}$ was zero (Fig. 4). Otherwise, $\sigma_{P(m=k)} / \sigma_{P(m=1)}$ was greater than $1 / \sqrt{ } k$. In the case of $\mathrm{CV}_{a}$ being $0.42(>0), \sigma_{P(m=k)} / \sigma_{P(m=1)}$ was always greater than $1 / \sqrt{ } k$. The conditions of Eq. (19) were thus never satisfied, which supports our hypothesis.

$\sigma_{P(m=k)} / \sigma_{P(m=1)}$ was near to 1.0 (i.e., $\sigma_{P(m=k)}$ was near to $\left.\sigma_{P(m=1)}\right)$, when $\mathrm{CV}_{<F>}$ was large. Measuring $F$ for two or more azimuthal directions thus hardly reduced the variation in $\langle F\rangle_{i, m}^{*} \cdot a_{i}^{*}$, when the variation in $\langle F\rangle^{*}{ }_{i, m=\infty}$ was large (i.e., the variation in the distribution of $F_{i, j}^{*}$ among trees was large). 

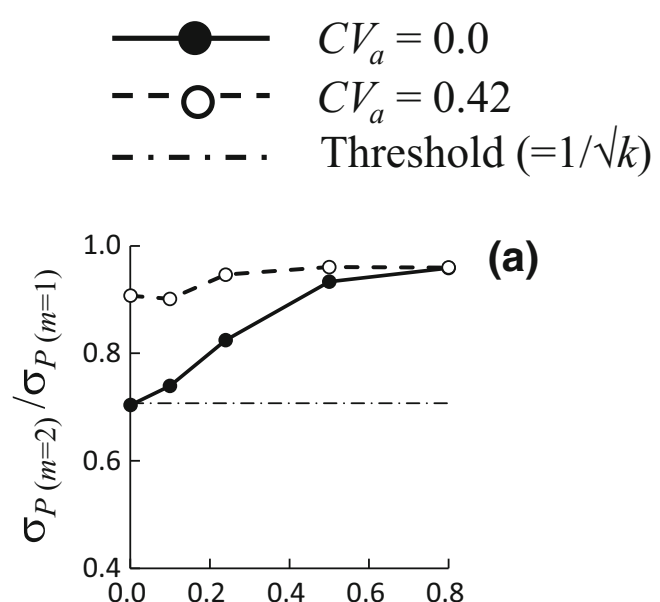

(a)

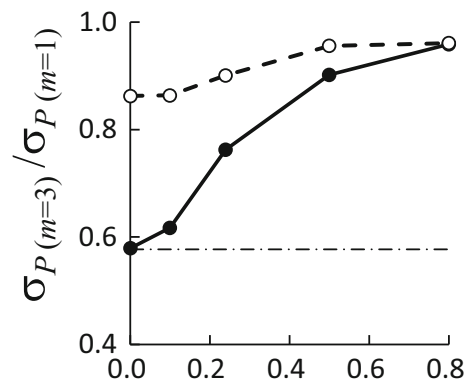

(b)

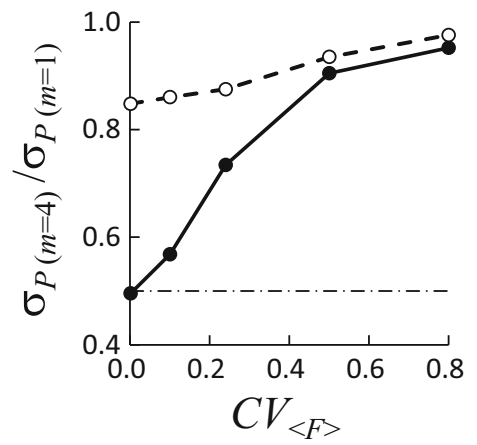

(c)

Fig. 4 Changes in $\sigma_{P(m=k)} / \sigma_{P(m=1)}$ with changing between-tree variations in sap flux density $\left(\mathrm{CV}_{<F>}\right)$ when between-tree variations in sapwood area $\left(\mathrm{CV}_{a}\right)$ were zero and $0.42 . k$ was assumed as a 2 , b 3 , and $\mathbf{c} 4$

This suggests that measuring $F$ for two or more azimuthal directions hardly contributed to more reliable $E$ estimates particularly under these conditions, even when the number of sensors was not limited.

\section{Discussion and conclusions}

Our previous study identified the conditions under which measuring $F$ for two or more azimuthal directions reduces uncertainty in $E$ estimates more effectively than measuring $F$ for one azimuthal direction when the number of sensors is limited. This study, based on non-dimensionalization of the theory presented in our previous study, demonstrated that the conditions are never satisfied. That is, measuring $F$ for one azimuthal direction is always found to allow more reliable $E$ estimates within the framework of the theory, i.e., when azimuthal variations and between-tree variations in $F$ are not particularly systematic. Note that this conclusion is not sitespecific but applicable to any site, because our results did not qualitatively change with the changes in parameters. We reached this finding because we succeeded in nondimensionalization of the theory which allowed us more comprehensive treatment of the question and development of a general hypothesis.

In the numerical experiments, we arbitrarily assumed that $\langle F\rangle^{*}{ }_{i, m=\infty}$ and $a^{*}{ }_{i}$ followed a normal distribution. Readers might argue that these assumptions affect our results. However, we confirmed that changing the shape of the distributions did not change our results qualitatively. In other words, the conditions of Eq. (19) were again never satisfied in this case. We also confirmed that although we determined the parameters somewhat arbitrarily, this parameterization did not change our results. We even confirmed that assuming variations in $\mathrm{CV}_{F}$ among the trees (not assuming a constant value for all trees) did not change our results qualitatively. These results were corollary of that our hypothesis was derived without making any specific assumptions about parameters used.

Based on the results, we conclude that it is unnecessary to examine whether the conditions are satisfied based on preliminary measurements of $F$ for the target site to determine the optimal allocation of sensors. If you have a good reason from results of previous studies to assume that azimuthal variations in $F$ would not be systematic for the target site, you can allocate sensors to measure $F$ for one azimuthal direction to cover between-tree variations in $F$. We here note that the one azimuthal direction for $F$ measurements should be determined randomly for each tree at the site. This is because there might be a tendency of higher/lower $F$ for specific azimuthal directions, which is too weak to be modeled in a systematic manner but still introduces possible biases in the $E$ estimate (see Komatsu et al. 2016). If you are uncertain whether or not azimuthal variations in $F$ are systematic for the target site, we recommend making preliminary measurements to confirm the assumption that there are no systematic azimuthal variations in $F$ before full installation of the sensors. We presume that azimuthal variations in $F$ are not very systematic in many cases, since the majority of previous studies reported no systematic azimuthal variations in $F$ (Lu et al. 2000; Cohen et al. 2008; Tateishi et al. 2008; Sato et al. 2012). This might not be the case for species/sites in which crown architecture is very skewed (e.g., a site located on a slope) and/or the effect of crown structure on xylem sap flow is particularly strong (see discussion in Oren et al. 1999 and López-Bernal et al. 2010). We are not saying that measuring azimuthal variations in $F$ is meaningless in most cases. Although researchers have not 
found regularity of azimuthal variations in $F$ in many cases, this might be attributable to limited information on azimuthal variations in $F$ and anatomical, structural, and ecophysiological traits of target trees. We thus advocate measuring azimuthal variations in $F$ and relevant anatomical, structural, and ecophysiological parameters under various conditions. Our point in this paper is that if you are interested solely in stand transpiration estimates and you are limited with the number of sensors, measuring $F$ for one random azimuthal direction is a good choice as long as no systematic azimuthal variation in $F$ is expected.

Acknowledgements We express our sincere thanks to Prof. Ram Oren (Duke University, USA) for providing critical comments on an earlier draft of this paper. Thanks are also due to Prof. Erwin Dreyer (INRA, France) and two anonymous reviewers for their helpful comments.

\section{Appendix}

Determination of parameters We determined parameters using data of $F$ and $a$ recorded for a 55-year-old $C$. japonica stand $\left(36^{\circ} 25^{\prime} \mathrm{N}, 136^{\circ} 38^{\prime} \mathrm{E}, 170 \mathrm{~m}\right.$ a.s.l.). A plot having an area of $300 \mathrm{~m}^{2}$ and containing 18 trees was established in the stand. The mean diameter at breast height of the 18 trees was $48.4 \mathrm{~cm}$ and the mean tree height was $27.9 \mathrm{~m}$. The sapwood area of the plot, determined from measurements of sapwood thickness using wood cores extracted from the trunks, was $36.6 \mathrm{~m}^{2} \mathrm{ha}^{-1}$. We used data for $F$ in four azimuthal directions (north, east, west, and south) recorded on nine of the 18 trees on seven sunny days in the period spanning 16 May to 4 June 2010 (16-18 May and 1-4 June). Measurements of $F$ were made using the heat-dissipation method with Graniertype sensors having a length of $20 \mathrm{~mm}$ (Granier 1987). Typically, two sensors were installed radially in each azimuthal direction to cover the sapwood depth and to measure radial variations. A complete description of the plot and measurements was given by Shinohara et al. (2013) and Komatsu et al. (2016).

The theory used in this study needed data for $F$ representative of each azimuthal direction after taking radial variations into account. We estimated $F$ representative of each azimuthal direction with the input of $F$ for the outmost xylem band of the direction. $F$ representative of an azimuthal direction was expressed by the mean of $F$ for the outmost and inner xylem bands weighted by circumference areas corresponding to the xylem bands (see Komatsu et al. 2016). We assumed that $a_{i}$ was identical to the circumference area on which sensors were installed. This assumption was based on the fact that Graniertype sensors measure the mean sap flux of the area where the sensors are attached (Granier 1987). $a_{i}$ values used here thus differed from those determined from data for sapwood thickness. However, the values were generally very similar; $a_{i}$ values for the nine trees used here were closely related to those determined from data for sapwood thickness (slope of 0.993 and $r^{2}=0.972$ ).

To determine $\mathrm{CV}_{F}$, we first calculated the $\mathrm{CV}$ of $F$ in four directions for each of the nine trees. Here, $F$ is a daily value averaged over the seven sunny days. We then calculated the mean value of the CVs for the nine trees and assumed this value to be $\mathrm{CV}_{F}$. To determine $\mathrm{CV}_{<F>}$, we first calculated the mean value of $F$ in four directions for each of the nine trees. We assumed the $\mathrm{CV}$ of these mean values of $F$ for the nine trees to be $\mathrm{CV}_{<F>} . \mathrm{CV}_{a}$ was assumed to equal the $\mathrm{CV}$ of $a_{i}$ among the nine trees.

\section{References}

Alsheimer M, Köstner B, Falge E, Tenhunen JD (1998) Temporal and spatial variation in transpiration of Norway spruce stands within a forested catchment of the Fichtelgebirge, Germany. Ann Sci For 55: 103-123. doi:10.1051/forest:19980107

Bond-Lamberty B, Wang C, Gower ST (2002) Aboveground and belowground biomass and sapwood area allometric equations for six boreal tree species of northern Manitoba. Can J For Res 32:14411450. doi: $10.1139 / \mathrm{x} 02-063$

Bosch DD, Marshall LK, Teskey R (2015) Forest transpiration from sap flux density measurements in a southeastern coastal plain riparian buffer system. Agric For Meteorol 187:72-82. doi:10.1016/j. agrformet.2013.12.002

Cohen Y, Cohen S, Cantuarias-Aviles T, Schiller G (2008) Variations in the radial gradient of sap velocity in trunks of forest and fruit trees. Plant Soil 305:49-59. doi:10.1007/s11104-007-9351-0

Delzon S, Loustau D (2005) Age-related decline in stand water use: sap flow and transpiration in a pine forest chronosequence. Agric For Meteorol 129:105-119. doi:10.1016/j.agrformet.2005.01.002

Ewers BE, Oren R (2000) Analyses of assumptions and errors in the calculation of stomatal conductance from sap flux measurements. Tree Physiol 20:579-589. doi:10.1093/treephys/20. 9.579

Ewers BE, Gower ST, Bond-Lamberty B, Wang CK (2005) Effects of stand age and tree species composition on transpiration and canopy conductance of boreal forest stands. Plant Cell Environ 28:660-678. doi:10.1111/j.1365-3040.2005.01312.x

Ewers BE, Bond-Lamberty B, Mackay S (2011) Consequences of stand age and species' functional trait changes on ecosystem water use of forests. In: Meinzer FC, Lachenbruch B, Dawson TE (eds) Size- and age-related changes in tree structure and function. Springer, Dordrecht, pp 481-505. doi:10.1007/978-94-007-1242-3 18

Ford CR, Hubbard RM, Kloeppel BD, Vose JM (2007) A comparison of sap flux-based evapotranspiration estimates with catchment-scale water balance. Agric For Meteorol 145:176-185. doi:10.1016/j. agrformet.2007.04.010

Fujii H (2005) Practical methods of data analysis for engineers. TokyoKagaku-Dojin, Tokyo

Granier A (1987) Evaluation of transpiration in a Douglas-fir stand by means of sap flow measurements. Tree Physiol 3:309-320. doi:10. 1093/treephys/3.4.309

Granier A, Biron P, Köstner B, Gay LW, Najjar G (1996) Comparisons of xylem sap flow and water vapour flux at the stand level and derivation of canopy conductance for Scots pine. Theor Appl Climatol 53: 115-122. doi:10.1007/BF00866416 
Granier A, Biron P, Lemoine D (2000) Water balance, transpiration and canopy conductance in two beech stands. Agric For Meteorol 100: 291-308. doi:10.1016/S0168-1923(99)00151-3

Komatsu H, Onozawa Y, Kume T, Tsuruta K, Kumagai T, Shinohara Y, Otsuki K (2010) Stand-scale transpiration estimates in a Moso bamboo forest: (II) comparison with coniferous forests. For Ecol Manag 260:1295-1302. doi:10.1016/j.foreco.2010.06.040

Komatsu H, Onozawa Y, Kume T, Tsuruta K, Shinohara Y, Otsuki K (2012) Canopy conductance for a Moso bamboo (Phyllostachys pubescens) forest in western Japan. Agric For Meteorol 156:111120. doi:10.1016/j.agrformet.2012.01.004

Komatsu H, Shinohara Y, Kumagai T, Kume T, Tsuruta K, Xiang Y, Ichihashi R, Tateishi M, Shimizu T, Miyazawa Y, Nogata M, Laplace S, Han T, Chiu CW, Ogura A, Saito T, Otsuki K (2014) A model relating transpiration for Japanese cedar and cypress plantations with stand structure. For Ecol Manag 334:301-312. doi:10. 1016/j.foreco.2014.08.041

Komatsu H, Shinohara Y, Kume T, Tsuruta K, Otsuki K (2016) Does measuring azimuthal variations in sap flux lead to more reliable stand transpiration estimates? Hydrol Process 30:2129-2137. doi: 10.1002/hyp. 10780

Köstner B, Biron P, Siegwolf R, Granier A (1996) Estimates of water vapor flux and canopy conductance of Scots pine at the tree level utilizing different xylem sap flow methods. Theor Appl Climatol 53: 105-113. doi:10.1007/BF00866415

Kumagai T, Nagasawa H, Mabuchi T, Ohsaki S, Kubota K, Kogi K, Utsumi Y, Koga S, Otsuki K (2005) Sources of error in estimating stand transpiration using allometric relationships between stem diameter and sapwood area for Cryptomeria japonica and Chamaecyparis obtusa. For Ecol Manag 206:191-195. doi:10. 1016/j.foreco.2004.10.066

Kumagai T, Aoki S, Shimizu T, Otsuki K (2007) Sap flow estimates of stand transpiration at two slope positions in a Japanese cedar forest watershed. Tree Physiol 27:161-168. doi:10.1093/treephys/27.2. 161

Kume T, Tsuruta K, Komatsu H, Kumagai T, Higashi N, Shinohara Y, Otsuki K (2010a) Effects of sample size on sap flux-based standscale transpiration estimates. Tree Physiol 30:129-138. doi:10. 1093/treephys/tpp074

Kume T, Onozawa Y, Komatsu H, Tsuruta K, Shinohara Y, Umebayashi T, Otsuki K (2010b) Stand-scale transpiration estimates in a Moso bamboo forest: (I) applicability of sap flux measurements. For Ecol Manag 260:1287-1294. doi:10.1016/j.foreco.2010.07.012

Kume T, Tanaka N, Kuraji K, Komatsu H, Yoshifuji N, Saitoh TM, Suzuki M, Kumagai T (2011) Ten-year evapotranspiration estimates in a Bornean tropical rainforest. Agric For Meteorol 151:11831192. doi:10.1016/j.agrformet.2011.04.005

Kume T, Otsuki K, Du S, Yamanaka N, Wang Y, Liu G (2012) Spatial variation in sap flow velocity in semiarid region trees: its impact on stand-scale transpiration estimates. Hydrol Process 26:1161-1168. doi:10.1002/hyp. 8205

Kume T, Tsuruta K, Komatsu H, Shinohara Y, Katayama A, Ide J, Otsuki K (2016) Differences in sap flux based stand transpiration between upper and lower slope positions in a Japanese cypress plantation watershed. Ecohydrology 9:1105-1116. doi:10.1002/eco.1709

Landsberg JJ, Waring RH (1997) A generalised model of forest productivity using simplified concepts of radiation-use efficiency, carbon balance and partitioning. For Ecol Manag 95:209-228. doi:10.1016/ S0378-1127(97)00026-1

Leverenz J, Deans JD, Ford ED, Jarvis PG, Milne R, Whitehead D (1982) Systematic spatial variation of stomatal conductance in a Sitka spruce plantation. J Appl Ecol 19:835-851. doi:10.2307/2403286

López-Bernal Á, Alcántara E, Testi L, Villalobos FJ (2010) Spatial sap flow and xylem anatomical characteristics in olive trees under different irrigation regimes. Tree Physiol 30:1536-1544. doi:10.1093/ treephys/tpq095
Loustau D, Domec JC, Bosc A (1998) Interpreting the variations in xylem sap flux density within the trunk of maritime pine (Pinus pinaster Ait.): application of a model for calculating water flows at tree and stand levels. Ann Sci For 55:29-46. doi:10.1051/forest:19980103

Lu P, Muller WJ, Chacko EK (2000) Spatial variation in xylem sap flux density in the trunk of orchard-grown, mature mango trees under changing soil water conditions. Tree Physiol 20:683-692. doi:10. 1093/treephys/20.10.683

Macfarlane C, Bond C, White DA, Grigg AH, Ogden GN, Silberstein R (2010) Transpiration and hydraulic traits of old and regrowth eucalypt forest in southwestern Australia. For Ecol Manag 260:96-105. doi:10.1016/j.foreco.2010.04.005

Moon M, Kim T, Park J, Cho S, Ryu D, Kim HS (2015) Variation in sap flux density and its effect on stand transpiration estimates of Korean pine stands. J For Res 20:85-93. doi:10.1007/s10310-014-0463-0

Oren R, Phillips N, Katul G, Ewers BE, Pataki DE (1998) Scaling xylem sap flux and soil water balance and calculating variance: a method for partitioning water flux in forests. Ann Sci For 55:191-216. doi: 10.1051/forest: 19980112

Oren R, Phillips N, Ewers BE, Pataki DE, Megonigal JP (1999) Sap flux scaled transpiration response to light, vapor pressure deficit, and leaf are reduction in a flooded Taxodium distichum forest. Tree Physiol 19:337-347. doi:10.1093/treephys/19.6.337

Pataki D, Oren R, Smith WK (2000) Sap flux of co-occuring species in a western subalpine forest during seasonal soil drought. Ecology 81: $2557-2566$

Peck R, Devore JL (2011) Statistics: the exploration and analysis of data. Brooks/Cole, Boston, Massachusetts

Poyatos R, Čermák J, Llorens P (2007) Variation in the radial patterns of sap flux density in pubescent oak (Quercus pubescens) and its implications for tree and stand transpiration measurements. Tree Physiol 27:537-548. doi:10.1093/treephys/27.4.537

Running SW, Coughlan JC (1988) A general model of forest ecosystem processes for regional applications. I. Hydrologic balance, canopy gas exchange and primary production processes. Ecol Model 42: 125-154. doi:10.1016/0304-3800(88)90112-3

Sato T, Oda T, Igarashi Y, Suzuki M, Uchiyama Y (2012) Circumferential sap flow variation in the trunks of Japanese cedar and cypress trees growing on a steep slope. Hydrol Res Lett 6:104-108. doi:10.3178/ HRL.6.104

Shinohara Y, Otsuki K (2016) Comparisons of soil-water content between a Moso bamboo (Phyllostachys pubescens) forest and an evergreen broadleaved forest in western Japan. Plant Spec Biol 30:96-103. doi:10.1111/1442-1984.12076

Shinohara Y, Tsuruta K, Ogura A, Noto F, Komatsu H, Otsuki K, Maruyama T (2013) Azimuthal and radial variations in sap flux density and effects on stand-scale transpiration estimates in a Japanese cedar forest. Tree Physiol 33:550-558. doi:10.1093/ treephys/tpt029

Swanson RH (1994) Significant historical developments in thermal methods for measuring sap flow in trees. Agric For Meteorol 72: 113-132. doi:10.1016/0168-1923(94)90094-9

Tateishi M, Kumagai T, Utsumi Y, Umebayashi T, Shiiba Y, Inoue K, Kaji K, Cho K, Otsuki K (2008) Spatial variations in xylem sap flux density in evergreen oak trees with radial-porous wood: comparisons with anatomical observations. Trees 22:23-30. doi:10.1007/ s00468-007-0165-8

Tsuruta T, Kume T, Komatsu H, Higashi N, Umebayashi T, Kumagai T, Otsuki K (2010) Azimuthal variations of sap flux density within Japanese cypress xylem trunks and their effects on tree transpiration estimates. J For Res 15:398-403. doi:10.1007/s10310-010-0202-0

Vertessy RA, Watson FGR, O'Sullivan SK (2001) Factors determining relations between stand age and catchment water balance in mountain ash forests. For Ecol Manag 143:13-26. doi:10.1016/S03781127(00)00501-6 